Research Article

\title{
New Exact Traveling Wave Solutions of the Time Fractional Complex Ginzburg-Landau Equation via the Conformable Fractional Derivative
}

\author{
Zhao Li $(\mathbb{D})$ and Tianyong Han \\ College of Computer Science, Chengdu University, Chengdu 610106, China \\ Correspondence should be addressed to Zhao Li; lizhao10.26@163.com
}

Received 30 September 2020; Revised 17 November 2020; Accepted 28 December 2020; Published 8 January 2021

Academic Editor: Ming Mei

Copyright ( 2021 Zhao Li and Tianyong Han. This is an open access article distributed under the Creative Commons Attribution License, which permits unrestricted use, distribution, and reproduction in any medium, provided the original work is properly cited.

\begin{abstract}
In this study, the exact traveling wave solutions of the time fractional complex Ginzburg-Landau equation with the Kerr law and dual-power law nonlinearity are studied. The nonlinear fractional partial differential equations are converted to a nonlinear ordinary differential equation via a traveling wave transformation in the sense of conformable fractional derivatives. A range of solutions, which include hyperbolic function solutions, trigonometric function solutions, and rational function solutions, is derived by utilizing the new extended $\left(G^{\prime} / G\right)$-expansion method. By selecting appropriate parameters of the solutions, numerical simulations are presented to explain further the propagation of optical pulses in optic fibers.
\end{abstract}

\section{Introduction}

The Ginzburg-Landau (GL) equation [1-7] is one of the most important partial differential equations in the field of mathematics and physics, which was introduced into the study of superconductivity phenomenology theory in the 20th century by Ginzburg and Landau. The GL equation usually describes the optical soliton [8-15] propagation through optical fibers over longer distances. Therefore, it is very important to analyze the dynamic behavior of the GL equation. In particular, searching for the approximate analytical solutions or exact traveling solutions of the GL equation has been more and more widely followed with interest because they can help to explain the complexity of nonlinear optics. So, in recent years, many powerful methods were used for finding the exact traveling solutions of the GL equation, which include the first integral method [16], dynamical system method [17], $\left(G^{\prime} / G\right)$-expansion method [18], Hirota's method [19], and harmonic balance method [20].

In recent years, the fractional GL equation [21-24] has been more and more widely followed with interest because it can be used to accurately model some nonlinear optical phenomena. In particular, constructing the exact traveling solutions of the fractional GL equation is very important work because it can be better explained the dynamics of soliton propagation through optical fibers over longer distances in nonlinear optics. So far, many important methods have been proposed to find the exact solutions of the fractional GL equation. In [25], Arshed extracted the hyperbolic, trigonometric, and rational function solutions of the fractional GL equation by the $\exp (-\phi(\xi))$-expansion method. In [26], Raza obtained the periodic and hyperbolic soliton solutions of the conformable time fractional GL equation by using the solitary wave ansatz method and $\exp (-\varphi(\chi))$-expansion method. In [27], Abdou et al. employed Jacobi's elliptic function expansion approach to retrieve doubly periodic function solutions for the time-space fractional GL equation. In [28], Sirisubtawee et al. investigated the cubic-quintic GL equation by the modified Kudryashov method and $\left(G^{\prime} / G, 1\right.$ $/ G)$-expansion method. In [29], Ghanbari and GómezAguilar obtained the periodic and hyperbolic soliton solutions of the conformable GL equation by using the generalized exponential rational function method. Although exact traveling wave solutions of the fractional GL equation have 
been successfully obtained by utilizing the above methods, it is far from enough on searching for the exact solution of a more general fractional complex GL equation. The main purpose of this paper is to construct exact traveling wave solutions of the time fractional complex GL equation with the Kerr law and dual-power law nonlinearity by using the new extended $\left(G^{\prime} / G\right)$-expansion method, and a range of solutions which include hyperbolic function solutions, trigonometric function solutions, rational function solutions, and negative power solutions is derived.

In this paper, we will consider the following time fractional complex GL equation [26, 29]:

$$
\begin{aligned}
i D_{t}^{\delta} u & +a u_{x x}+b F\left(|u|^{2}\right) u \\
& =\frac{\alpha|u|^{2}\left(|u|^{2}\right)_{x x}-\beta\left(\left(|u|^{2}\right)_{x}\right)^{2}}{|u|^{2} u^{*}}+\gamma u, \quad 0<\delta \leq 1,
\end{aligned}
$$

where $x$ represents distance across the fiber in dimensionless form. $a$ and $b$ denote the coefficient of the group velocity dispersion and the coefficient of nonlinearity. The terms with constants $\alpha$ and $\beta$ arise from the perturbation effects. In particular, $\gamma$ comes from the detuning effect. $a, b, \alpha, \beta$, and $\gamma$ are real-valued constants. $u^{*}$ is the complex conjugate numbers of $u$. Equation (1) is used to model various physical phenomena, such as nonlinear waves, Bose-Einstein condensation, second-order phase transitions, superconductivity, superfluidity, liquid crystals, and strings.

In Equation (1), $F\left(|u|^{2}\right) u: \mathbf{C} \rightarrow \mathbf{C}$ is a real-valued algebraic function. Let $F\left(|u|^{2}\right) u$ be $k$ times continuously differentiable. Then,

$$
F\left(|u|^{2}\right) u \in \bigcup_{m, n=1}^{\infty} C^{k}\left((-n, n) \times(-m, m) ; \mathbf{R}^{2}\right)
$$

where $\mathbf{C}$ is a complex plane of two-dimensional linear space in $\mathbf{R}^{2}$.

The rest of this paper is organized as follows. In Section 2, we will give the definition of the conformable fractional derivative and its properties. In Section 3, we will introduce the extended $\left(G^{\prime} / G\right)$-expansion method. In Section 4, we will discuss the exact traveling solutions of the fractional complex GL equation with the Kerr law and dual-power law nonlinearity by using the extended $\left(G^{\prime} / G\right)$-expansion method and plot the profiles of several representative exact traveling solutions. In Section 5, we will present the concluding remarks.

\section{Preliminaries}

Definition 1 [28]. Let $f:[0, \infty) \rightarrow \mathbf{R}$. Then, the conformable fractional derivative of $f$ of order $\alpha$ is defined as

$$
D_{t}^{\delta} f(t)=\lim _{\varepsilon \rightarrow 0} \frac{f\left(t+\varepsilon t^{1-\delta}\right)-f(t)}{\varepsilon}, \quad \text { for all } t>0,0<\delta \leq 1 \text {. }
$$

If the limit in Equation (3) exists, then we say that $f$ is $\delta$ -conformable differentiable at a point $t$.

Theorem 2 [28]. Let $\alpha \in(0,1]$ and $f(t)$ and $g(t)$ be $\delta$-conformable differentiable at a point $t>0$. Then,

$$
\begin{gathered}
D_{t}^{\delta}\left(t^{\mu}\right)=\mu t^{\mu-\delta}, \quad \text { for all } \mu \in \mathbf{R}, \\
D_{t}^{\delta}(a f(t)+b g(t))=a D_{t}^{\delta} f(t)+b D_{t}^{\delta} g(t), \quad \text { for all } a, b \in \mathbf{R}, \\
D_{t}^{\delta}(f(t) g(t))=f(t) D_{t}^{\delta} g(t)+g(t) D_{t}^{\delta} f(t), \\
D_{t}^{\delta}\left(\frac{f(t)}{g(t)}\right)=\frac{g(t) D_{t}^{\delta} f(t)-f(t) D_{t}^{\delta} g(t)}{g^{2}(t)}, \\
D_{t}^{\delta}(f(t))=t^{1-\delta} \frac{d f(t)}{d t}, \quad \text { provided that } f(t) \text { is differentiable. }
\end{gathered}
$$

Theorem 3 [28]. Let $f:(0, \infty) \rightarrow \mathbf{R}$ be a function such that $f$ is differentiable and $\delta$-conformable differentiable. Further, let $g$ be a differentiable function defined in the range of $f$. Then,

$$
D_{t}^{\alpha}(f \circ g)(t)=\left.t^{1-\alpha} g(t)^{\alpha-1} g^{\prime}(t) D_{t}^{\alpha}(f(t))\right|_{t=g(t)},
$$

where the prime notation $\left(^{\prime}\right)$ denotes the classical derivative.

\section{Overview of the Extended $\left(G^{\prime} / G\right)$-Expansion Method}

Let us consider a general fractional partial differential equation in the form

$$
P\left(u, u_{t}, u_{x}, \cdots, D_{t}^{\delta} u, D_{x}^{\delta} u, D_{t}^{\delta} D_{x}^{\delta}, \cdots\right)=0, \quad 0<\delta \leq 1,
$$

where $u$ is an unknown function and $P$ is a polynomial of $u$ and its partial fractional order derivatives, in which the highest order derivatives and nonlinear terms are involved.

Step 1. By using the traveling wave transformation $u(t, x)=$ $U(\xi)$, where $\xi=x-l\left(t^{\delta} / \delta\right), l$ is a nonzero arbitrary constant, $0<\delta \leq 1$, we can rewrite Equation (6) as the following nonlinear ordinary differential equation (ODE) of the integer order with respect to $\xi$ :

$$
Q\left(U, U^{\prime}, U^{\prime \prime}, \cdots\right)=0
$$

If it is possible, we should integrate Equation (7) term by term one or more times; the constants of integration are considered to be zero.

Step 2. Assume that the traveling wave solution of Equation (7) can be expressed as follows:

$$
U(\xi)=\sum_{i=0}^{N} a_{i}\left(\frac{G^{\prime}}{G}\right)^{i}+\sum_{i=1}^{N} b_{i}\left(\frac{G^{\prime}}{G}\right)^{-i}
$$


where either $a_{N}$ or $b_{N}$ may be zero, but both $a_{N}$ and $b_{N}$ cannot be zero at a time, and $a_{i}(i=0,1,2, \cdots, N)$ and $b_{i}$ $(i=1,2, \cdots, N)$ are arbitrary constants, where $G=G(\xi)$ satisfies the following nonlinear ODE:

$$
G G^{\prime \prime}=A G^{\prime 2}+B G G^{\prime}+C G^{2},
$$

where $A, B$, and $C$ are real parameters.

Step 3. The value of the positive integer $N$ in Equation (8) can be computed by using the homogeneous balance between the highest order nonlinear term and the highest order derivative in $U(\xi)$ appearing in Equation (8).

Step 4. Substituting Equations (8) and (9) into Equation (7) with the value of $N$ obtained in Step 3, we obtain polynomials in $\left(G^{\prime} / G\right)^{N}(N=0,1,2, \cdots)$ and $\left(G^{\prime} / G\right)^{-N}(N=0,1,2, \cdots)$.
Then, we collect all coefficients of the resulted polynomial to zero, and we obtain a set of algebraic equations for $a_{i}$ $(i=0,1,2, \cdots)$ and $b_{i}(i=1,2, \cdots, N)$.

Step 5. By solving the algebraic equations obtained in Step 4, we can obtain the values of the constants $a_{i}(i=0,1,2, \cdots)$ and $b_{i}(i=1,2, \cdots, N)$. Replacing the values into Equation (8), we construct a more general type and new exact traveling wave solutions of Equation (3).

The solutions of Equation (9) can be categorized into the following three cases.

Case 1 (hyperbolic function solutions). When $B^{2}-4(A-1)$ $C>0$ and $A \neq 1$, then

$$
\frac{G^{\prime}(\xi)}{G(\xi)}=\frac{\sqrt{B^{2}+4 C-4 A C}}{2(1-A)}\left(\frac{C_{1} \sinh \left(\left(\sqrt{B^{2}+4 C-4 A C} / 2\right) \xi\right)+C_{2} \cosh \left(\left(\sqrt{B^{2}+4 C-4 A C} / 2\right) \xi\right)}{C_{1} \cosh \left(\left(\sqrt{B^{2}+4 C-4 A C} / 2\right) \xi\right)+C_{2} \sinh \left(\left(\sqrt{B^{2}+4 C-4 A C} / 2\right) \xi\right)}\right)+\frac{B}{2(1-A)} .
$$

Case 2 (trigonometric function solutions). When $4(A-1) C$

$-B^{2}>0$ and $A \neq 1$, then

$$
\frac{G^{\prime}(\xi)}{G(\xi)}=\frac{\sqrt{4 A C-4 C-B^{2}}}{2(1-A)}\left(\frac{-C_{1} \sin \left(\left(\sqrt{4 A C-4 C-B^{2}} / 2\right) \xi\right)+C_{2} \cos \left(\left(\sqrt{4 A C-4 C-B^{2}} / 2\right) \xi\right)}{C_{1} \cos \left(\left(\sqrt{4 A C-4 C-B^{2}} / 2\right) \xi\right)+C_{2} \sin \left(\left(\sqrt{4 A C-4 C-B^{2}} / 2\right) \xi\right)}\right)+\frac{B}{2(1-A)}
$$

Case 3 (rational function solutions). When $B^{2}-4(A-1) C$ $=0$ and $A \neq 1$, then

$$
\frac{G^{\prime}(\xi)}{G(\xi)}=\frac{1}{1-A}\left(\frac{C_{1}}{C_{1} \xi+C_{2}}+\frac{B}{2}\right)
$$

Remark 4. In fact, by taking $A=0, B=-\lambda$, and $C=-\mu$ in Equation (9), the second nonlinear ODE reduces into the following second-order linear ODE:

$$
G^{\prime \prime}+\lambda G^{\prime}+\mu G=0
$$

\section{Exact Solutions of the Time Fractional Complex Ginzburg-Landau Equation}

In order to obtain the traveling wave solution of Equation (1), we assume that the exact solution has the following form:

$$
u(t, x)=U(\xi) e^{i \eta},
$$

where $\xi=x-v\left(t^{\delta} / \delta\right)$ and $\eta=-k x+w\left(t^{\delta} / \delta\right)+\theta$.
Substituting (14) into Equation (1), then collecting the real and imaginary parts, we get the following equations:

$$
\begin{aligned}
\text { Re }: & -w U+a\left(U^{\prime \prime}-k^{2} U\right)+b F\left(U^{2}\right) U=2(\alpha-2 \beta) \frac{\left(U^{\prime}\right)^{2}}{U} \\
& +2 \alpha U^{\prime \prime}+\gamma U,
\end{aligned}
$$

$$
\operatorname{Im}: v=-2 a k,
$$

where $U^{\prime \prime}=d^{2} U / d \xi^{2}$.

By taking $\alpha=2 \beta$, Equation (15) becomes

$$
-w U+a\left(U^{\prime \prime}-k^{2} U\right)+b F\left(U^{2}\right) U=2 \alpha U^{\prime \prime}+\gamma U .
$$

4.1. Kerr Law Nonlinearity. The Kerr law nonlinearity is the case when $F(U)=U$; then, Equation (17) reduces to

$$
(2 \alpha-a) U^{\prime \prime}-b U^{3}+\left(\gamma+w+a k^{2}\right) U=0 .
$$




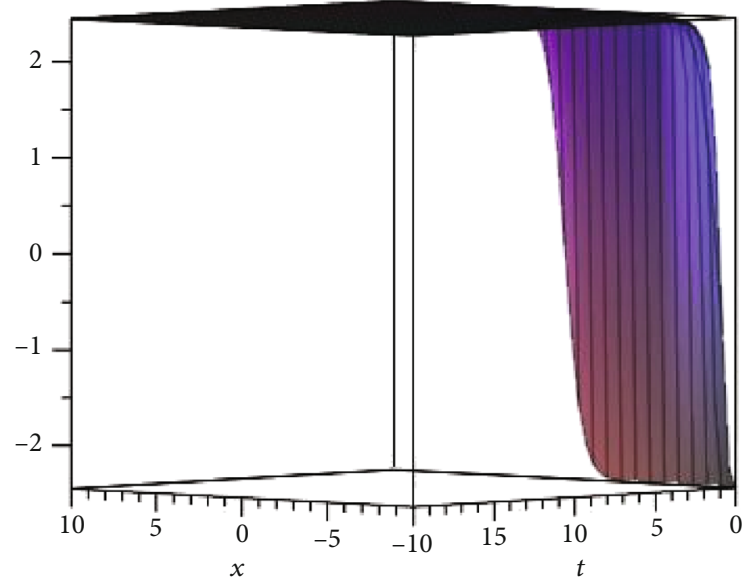

(a) $\delta=1 / 5$

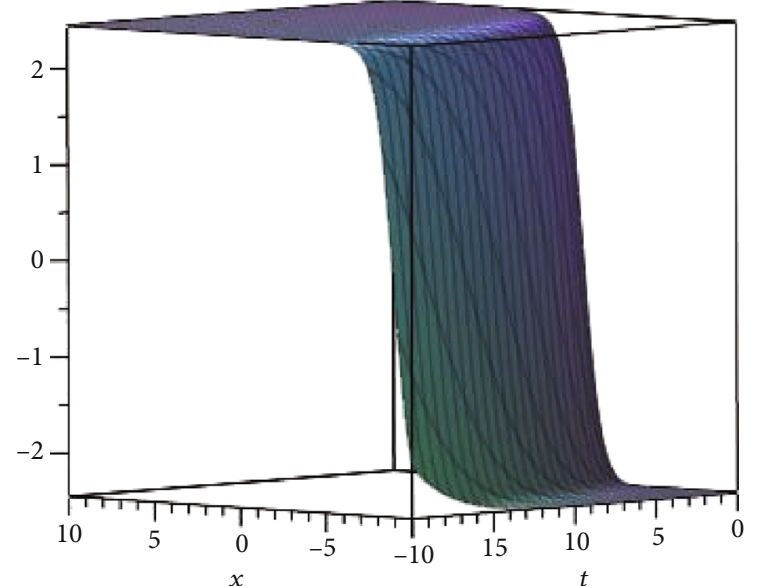

(b) $\delta=1 / 2$

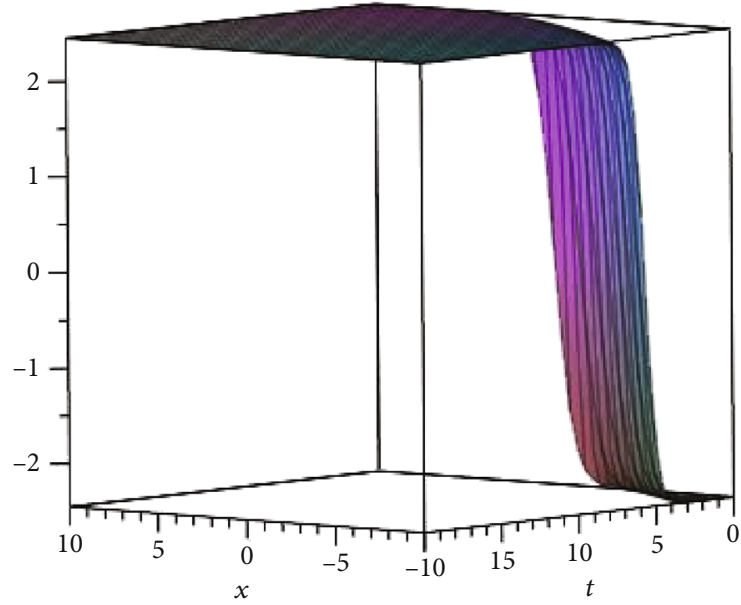

(c) $\delta=4 / 5$

FIgURE 1: The positive real part of $u_{1_{1}}(t, x)$ in Equation (23) for differential values of the fractional parameter $\delta$.

By using the homogeneous balance principle, we select $N=1$. Then, we suppose that the solution of Equation (18) satisfies

$$
U(\xi)=a_{1}\left(\frac{G^{\prime}}{G}\right)+a_{0}+b_{1}\left(\frac{G^{\prime}}{G}\right)^{-1},
$$

where $a_{1}, a_{0}$, and $b_{1}$ are constants to be determined.

Substituting (19) together with (9) into (18) yields a polynomial in $\left(G^{\prime} / G\right)^{N} \quad(N=0,1,2, \cdots)$ and $\left(G^{\prime} / G\right)^{-N}$ $(N=0,1,2, \cdots)$. Collecting the coefficient of the resulted polynomial and setting them to zero, we obtain a nonlinear algebraic equation in $a_{1}, a_{0}$, and $b_{1}$. Solving the algebraic equation using the computer algebraic software of Maple, we get the following results:

Case 1. $\quad a_{1}= \pm \sqrt{\left(2(2 \alpha-a)(A-1)^{2}\right) / b}, \quad a_{0}= \pm$ $\sqrt{\left((2 \alpha-a) B^{2}\right) / 2 b}, b_{1}=0, k=k$, and $w=\left((2 \alpha-a)\left(B^{2}+4 C\right.\right.$ $-4 A C)) / 2-\gamma-a k^{2}$.
Case 2. $\quad a_{1}=0, \quad a_{0}= \pm \sqrt{\left((2 \alpha-a) B^{2}\right) / 2 b}, \quad b_{1}= \pm$ $\sqrt{\left(2(2 \alpha-a) C^{2}\right) / b}, k=k$, and $w=\left(\left((2 \alpha-a)\left(B^{2}+4 C-4 A C\right.\right.\right.$ )$/ 2)-\gamma-a k^{2}$, where $A, B, C, a, b, \alpha$, and $\gamma$ are free parameters.

Substituting these solutions into (19), using (14), we obtain the traveling wave solution as follows:

$$
\begin{aligned}
& u(t, x)=\left[ \pm \sqrt{\frac{(2 \alpha-a) B^{2}}{2 b}} \pm \sqrt{\frac{2(2 \alpha-a)(A-1)^{2}}{b}}\left(\frac{G^{\prime}(\xi)}{G(\xi)}\right)\right] e^{i\left(-k x+w\left(t^{\delta} / \delta\right)+\theta\right),} \\
& u(t, x)=\left[ \pm \sqrt{\frac{(2 \alpha-a) B^{2}}{2 b}} \pm \sqrt{\frac{2(2 \alpha-a) C^{2}}{b}}\left(\frac{G^{\prime}(\xi)}{G(\xi)}\right)^{-1}\right] e^{i\left(-k x+w\left(t^{\delta} / \delta\right)+\theta\right),}
\end{aligned}
$$

where $G^{\prime}(\xi) / G(\xi)$ is defined in Section 3, and $\xi=x+2 a k$ $\left(t^{\delta} / \delta\right)$. 
Family 1. The hyperbolic function solutions can be obtained as $B^{2}-4(A-1) C>0$ and $A \neq 1$. Then,

$$
\begin{aligned}
u_{1}(t, x)= & {\left[ \pm \sqrt{\frac{(2 \alpha-a) B^{2}}{2 b}} \pm \sqrt{\frac{2(2 \alpha-a)(A-1)^{2}}{b}}\right.} \\
& \left.\cdot\left(\frac{B}{2(1-A)}+\frac{\sqrt{B^{2}+4 C-4 A C}}{2(1-A)} H_{1}\right)\right] e^{i\left(-k x+w\left(t^{\delta} / \delta\right)+\theta\right),} \\
u_{2}(t, x)= & {\left[ \pm \sqrt{\frac{(2 \alpha-a) B^{2}}{2 b}} \pm \sqrt{\frac{2(2 \alpha-a) C^{2}}{b}}\right.} \\
& \left.\cdot\left(\frac{B}{2(1-A)}+\frac{\sqrt{B^{2}+4 C-4 A C}}{2(1-A)} H_{1}\right)^{-1}\right] e^{i\left(-k x+w\left(t^{\delta} / \delta\right)+\theta\right),}
\end{aligned}
$$

where $H_{1}=\left(C_{1} \sinh \left(\left(\sqrt{B^{2}+4 C-4 A C} / 2\right)\left(x+2 a k\left(t^{\delta} / \delta\right)\right)\right)\right.$ $\left.+C_{2} \cosh \left(\left(\sqrt{B^{2}+4 C-4 A C} / 2\right) \quad\left(x+2 a k\left(t^{\delta} / \delta\right)\right)\right)\right) /$ $\left(C_{1} \cosh \left(\left(\sqrt{B^{2}+4 C-4 A C} / 2\right) \quad\left(x+2 a k\left(t^{\delta} / \delta\right)\right)\right)+C_{2} \sinh \right.$ $\left.\left(\left(\sqrt{B^{2}+4 C-4 A C} / 2\right)\left(x+2 a k\left(t^{\delta} / \delta\right)\right)\right)\right)$, and $C_{1}$ and $C_{2}$ are arbitrary constants.

In particular, if we assume that $C_{2}=0, A>1, B>0$, and $C_{1} \neq 0$ in (21), then we have

$$
\begin{aligned}
u_{1_{1}}(t, x)= & \mp \sqrt{\frac{(2 \alpha-a)\left(B^{2}+4 C-4 A C\right)}{2 b}}\left[\operatorname { t a n h } \left(\frac{\sqrt{B^{2}+4 C-4 A C}}{2}\right.\right. \\
& \left.\left.\cdot\left(x+2 a k\left(t^{\delta} / \delta\right)\right)\right)\right] e^{i\left(-k x+w\left(t^{\delta} / \delta\right)+\theta\right)},
\end{aligned}
$$

and if $C_{1}=0, A>1, B>0$, and $C_{2} \neq 0$ in (21), then we obtain

$$
\begin{aligned}
u_{1_{2}}(t, x)= & \mp \sqrt{\frac{(2 \alpha-a)\left(B^{2}+4 C-4 A C\right)}{2 b}}\left[\operatorname { c o t h } \left(\frac{\sqrt{B^{2}+4 C-4 A C}}{2}\right.\right. \\
& \left.\left.\cdot\left(x+2 a k\left(t^{\delta} / \delta\right)\right)\right)\right] e^{i\left(-k x+w\left(t^{\delta} / \delta\right)+\theta\right)} .
\end{aligned}
$$

The fixed values are $\alpha=2, a=1, b=1, A=2, B=4, C=3$, and $k=1$, and the fractional parameter $\delta \in\{1 / 5,1 / 2,1\}$ is used to plot the positive real part of the exact solution $u_{1_{1}}(t, x)$ as in Figures 1 and 2.

Family 2. The trigonometric function solutions can be obtained as $4(A-1) C-B^{2}>0$ and $A \neq 1$. Then,

$$
\begin{aligned}
u_{3}(t, x)= & {\left[ \pm \sqrt{\frac{(2 \alpha-a) B^{2}}{2 b}} \pm \sqrt{\frac{2(2 \alpha-a)(A-1)^{2}}{b}}\left(\frac{B}{2(1-A)}\right.\right.} \\
& \left.\left.+\frac{\sqrt{4 A C-4 C-B^{2}}}{2(1-A)} H_{2}\right)\right] e^{i\left(-k x+w\left(t^{\delta} / \delta\right)+\theta\right)},
\end{aligned}
$$

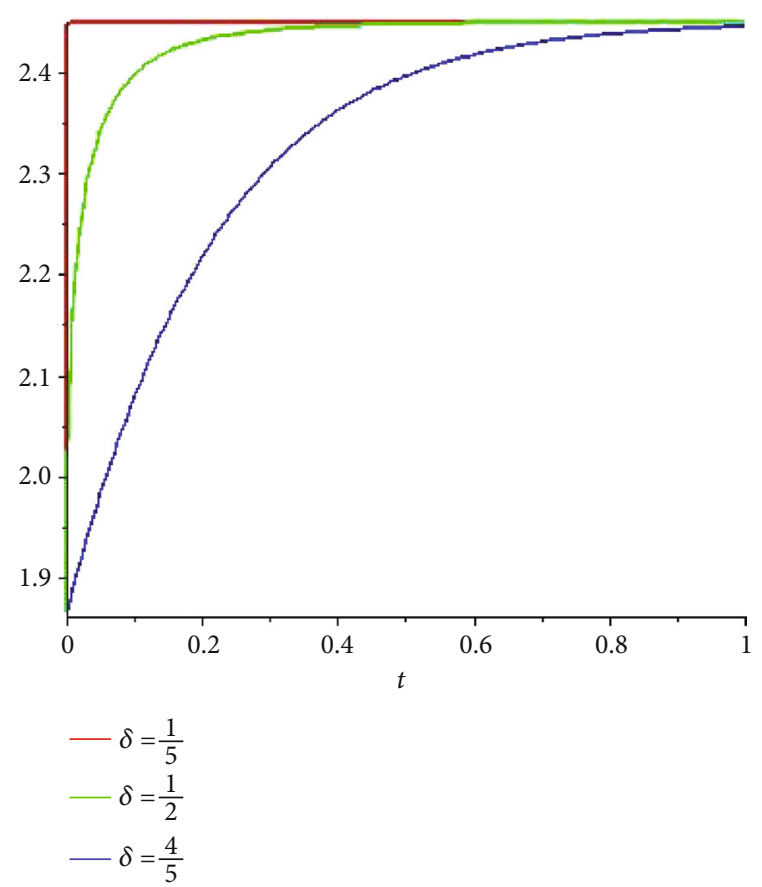

FIgURE 2: The positive real part of $u_{1_{1}}(t, x)$ at $x=1.0$ in Equation (23) for differential values of the fractional parameter $\delta$.

$$
\begin{aligned}
u_{4}(t, x)= & {\left[ \pm \sqrt{\frac{(2 \alpha-a) B^{2}}{2 b}} \pm \sqrt{\frac{2(2 \alpha-a) C^{2}}{b}}\right.} \\
& \left.\cdot\left(\frac{B}{2(1-A)}+\frac{\sqrt{4 A C-4 C-B^{2}}}{2(1-A)} H_{2}\right)^{-1}\right] e^{i\left(-k x+w\left(t^{\delta} / \delta\right)+\theta\right),}
\end{aligned}
$$

where $H_{2}=\left(-C_{1} \sin \left(\left(\sqrt{4 A C-B^{2}-4 C} / 2\right)\left(x+2 a k\left(t^{\delta} / \delta\right)\right)\right)\right.$ $\left.+C_{2} \cos \left(\left(\sqrt{4 A C-B^{2}-4 C} / 2\right)\left(x+2 a k\left(t^{\delta} / \delta\right)\right)\right)\right) / \quad\left(C_{1}\right.$ $\cos \left(\left(\sqrt{4 A C-B^{2}-4 C} / 2\right)\left(x+2 a k\left(t^{\delta} / \delta\right)\right)\right)+C_{2} \sin (($ $\left.\left.\left.\sqrt{4 A C-B^{2}-4 C} / 2\right)\left(x+2 a k\left(t^{\delta} / \delta\right)\right)\right)\right)$, and $C_{1}$ and $C_{2}$ are arbitrary constants.

Specifically, if we suppose that $C_{2}=0, A>1, B>0$, and $C_{1} \neq 0$ in (25), then we obtain

$$
\begin{aligned}
u_{3_{1}}(t, x)= & \pm \sqrt{\frac{(2 \alpha-a))\left(4 A C-4 C-B^{2}\right)}{2 b}}\left[\operatorname { t a n } \left(\frac{\sqrt{4 A C-4 C-B^{2}}}{2}\right.\right. \\
& \left.\left.\cdot\left(x+2 a k \frac{t^{\delta}}{\delta}\right)\right)\right] e^{i\left(-k x+w\left(t^{\delta} / \delta\right)+\theta\right)},
\end{aligned}
$$

and if $C_{1}=0, A>1, B>0$, and $C_{2} \neq 0$ in (25), then we have

$$
\begin{aligned}
u_{3_{2}}(t, x)= & \mp \sqrt{\frac{(2 \alpha-a)\left(4 A C-4 C-B^{2}\right)}{2 b}}\left[\operatorname { c o t } \left(\frac{\sqrt{4 A C-4 C-B^{2}}}{2}\right.\right. \\
& \left.\left.\cdot\left(x+2 a k \frac{t^{\delta}}{\delta}\right)\right)\right] e^{i\left(-k x+w\left(t^{\delta} / \delta\right)+\theta\right)} .
\end{aligned}
$$




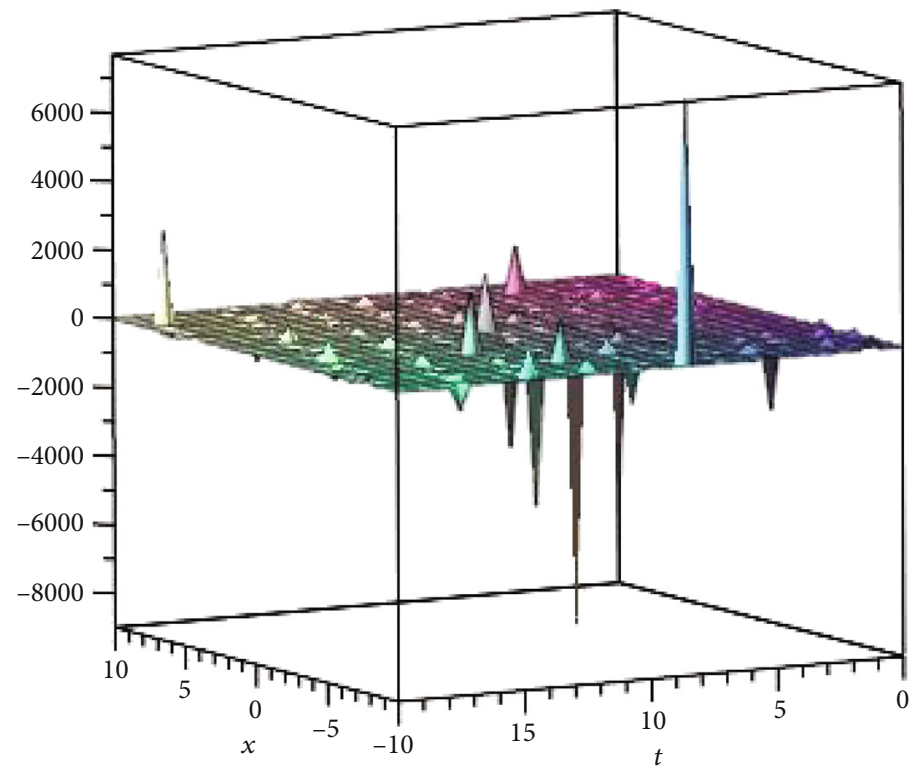

(a) $\delta=1 / 5$

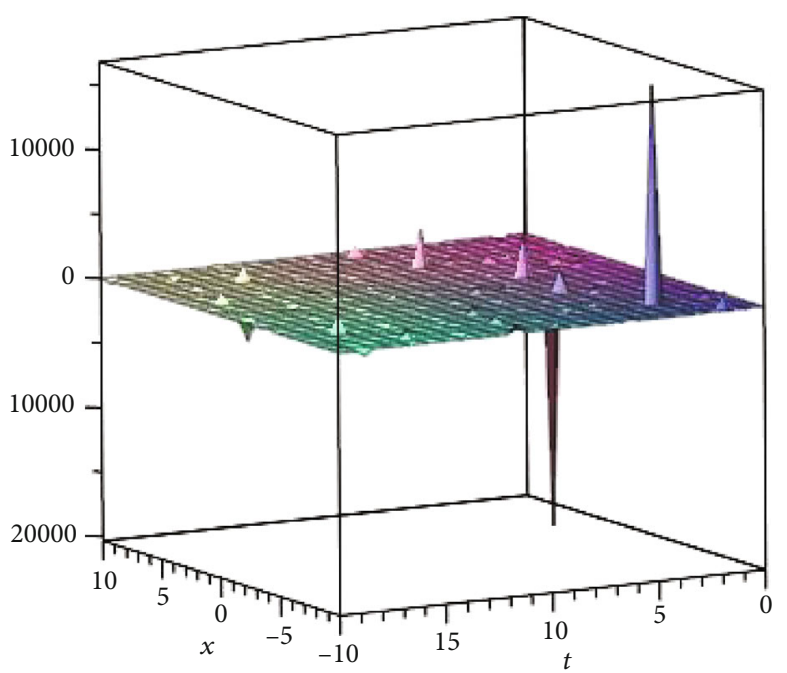

(b) $\delta=1 / 2$

FIgURE 3: The positive real part of $u_{3_{1}}(t, x)$ in Equation (27) for differential values of the fractional parameter $\delta$.

The fixed values are $\alpha=2, a=1, b=1, A=4, B=3, C=3$, and $k=1$, and the fractional parameter $\delta \in\{1 / 5,1 / 2,4 / 5\}$ is used to plot the positive real part of the exact solution $u_{3_{1}}(t, x)$ as in Figures 3 and 4 .

Family 3. The rational function solutions can be obtained as $B^{2}-4(A-1) C=0$ and $A \neq 1$. Then,

$$
\begin{aligned}
u_{5}(t, x)= & {\left[ \pm \sqrt{\frac{(2 \alpha-a) B^{2}}{2 b}} \pm \frac{1}{1-A} \sqrt{\frac{2(2 \alpha-a)(A-1)^{2}}{b}}\right.} \\
& \left.\cdot\left(\frac{C_{1}}{C_{1}\left(x+2 a k\left(t^{\delta} / \delta\right)\right)+C_{2}}+\frac{B}{2}\right)\right] e^{i\left(-k x+w\left(t^{\delta} / \delta\right)+\theta\right)},
\end{aligned}
$$

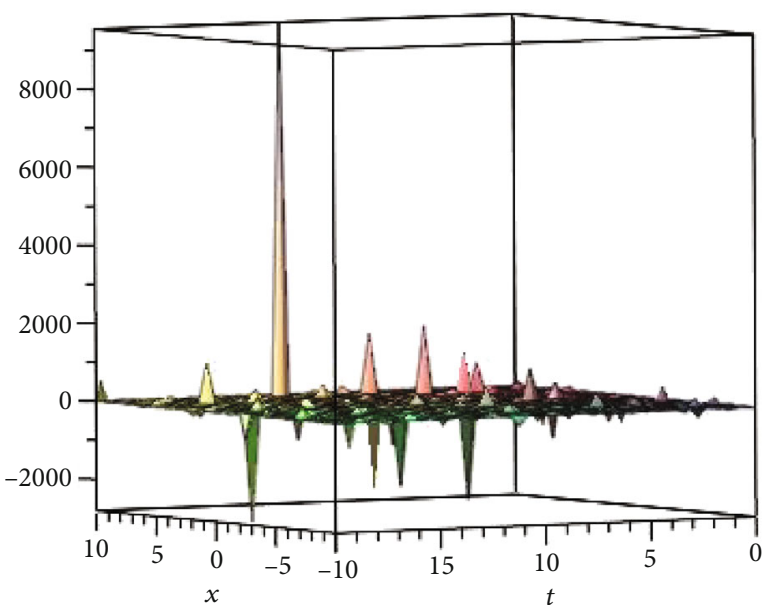

(c) $\delta=4 / 5$

$$
\begin{aligned}
u_{6}(t, x)= & {\left[ \pm \sqrt{\frac{(2 \alpha-a) B^{2}}{2 b}} \pm \frac{1}{1-A} \sqrt{\frac{2(2 \alpha-a) C^{2}}{b}}\right.} \\
& \left.\cdot\left(\frac{C_{1}}{C_{1}\left(x+2 a k\left(t^{\delta} / \delta\right)\right)+C_{2}}+\frac{B}{2}\right)^{-1}\right] e^{i\left(-k x+w\left(t^{\delta} / \delta\right)+\theta\right)},
\end{aligned}
$$

where $C_{1}$ and $C_{2}$ are arbitrary constants.

4.2. Dual-Power Law. In this case, $F(U)=U^{n}+l U^{2 n}$; then, Equation (17) takes the following form:

$$
(2 \alpha-a) U^{\prime \prime}+\left(\gamma+w+a k^{2}\right) U-b\left(U^{2 n+1}+l U^{4 n+1}\right)=0,
$$

where $l$ is a constant. 


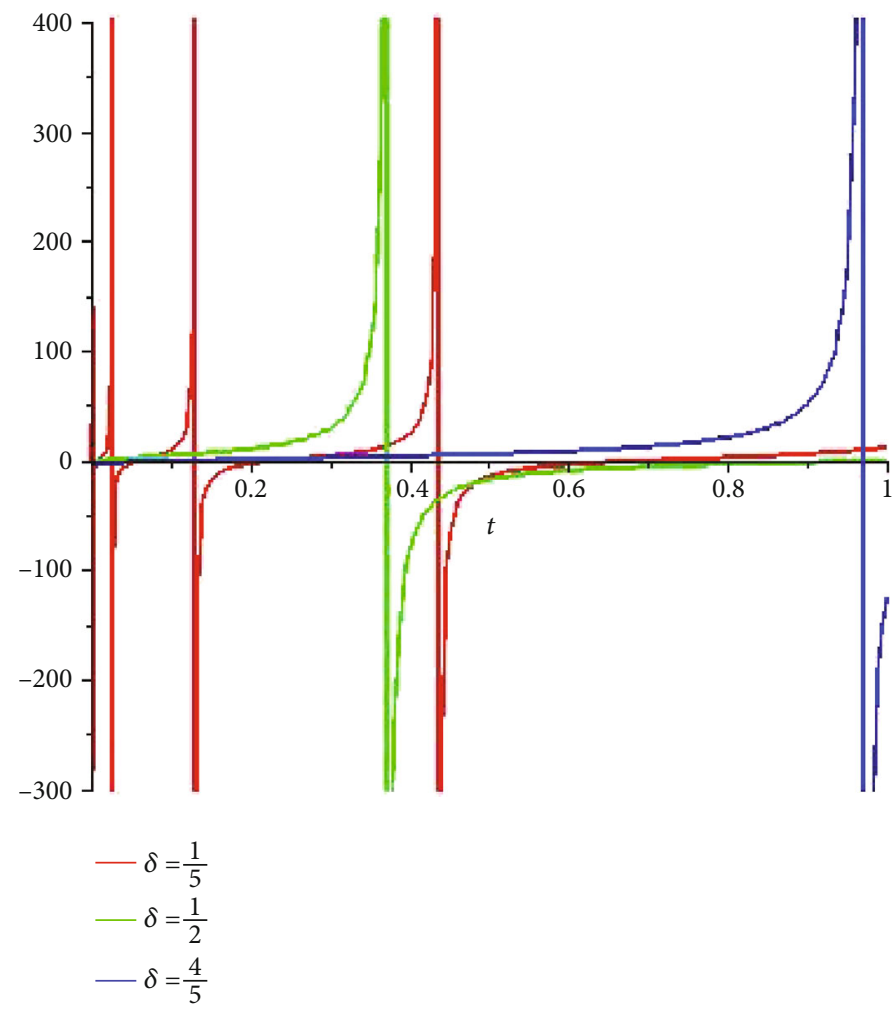

FIgURE 4: The positive real part of $u_{3_{1}}(t, x)$ at $x=1.0$ in Equation (27) for differential values of the fractional parameter $\delta$

Using transformation

$$
U=V^{1 / 2 n}
$$

Equation (30) will be reduced into the following ODE:

$$
\begin{aligned}
& (2 \alpha-a)\left[(1-2 n) V^{\prime 2}+2 n V V^{\prime \prime}\right]+4 n^{2}\left(\gamma+w+a k^{2}\right) V^{2} \\
& -4 b n^{2} V^{2}\left(V+l V^{2}\right)=0 .
\end{aligned}
$$

Now, applying the balancing principle between $V^{4}$ and $V V^{\prime \prime}$ in the above equation, we get $N=1$. Therefore, we have

$$
V(\xi)=a_{1}\left(\frac{G^{\prime}}{G}\right)+a_{0}+b_{1}\left(\frac{G^{\prime}}{G}\right)^{-1},
$$

where $a_{1}, a_{0}$, and $b_{1}$ are constants to be determined.

Substituting (33) together with (9) into (32) yields a polynomial in $\left(G^{\prime} / G\right)^{N} \quad(N=0,1,2, \cdots)$ and $\left(G^{\prime} / G\right)^{-N}$ $(N=0,1,2, \cdots)$. Collecting the coefficient of the resulted polynomial and setting them to zero, we obtain a nonlinear algebraic equation in $a_{1}, a_{0}$, and $b_{1}$. Solving the algebraic equation using the computer algebraic software of Maple, we get the following results:

Case 1. $a_{1}= \pm(1 / 2 n) \sqrt{\left((2 n+1)(2 \alpha-a)(A-1)^{2}\right) / b l}, a_{0}$ $=-((2 n+1) /(4 l(n+1))) \pm(B /(4 n(A-1)))$ $\sqrt{\left((2 n+1)(2 \alpha-a)(A-1)^{2}\right) / b l}, b_{1}=0, k=k$, and $w=(((2 \alpha$ $\left.\left.-a)\left(B^{2}+4 C-4 A C\right)\right) / 8 n^{2}\right)-\left((3 b(2 n+1)) /\left(8 l(n+1)^{2}\right)\right)-$ $\gamma-a k^{2}$.

Case 2. $a_{1}=0, a_{0}=-((2 n+1) /(4 l(n+1))) \pm(B / 4 n$ C) $\sqrt{\left((2 n+1)(2 \alpha-a) C^{2}\right) / b l}, \quad b_{1}= \pm(1 / 2 n)$ $\sqrt{\left(\left((2 n+1)(2 \alpha-a) C^{2}\right) / b l\right)}, k=k$, and $w=\left(\left((2 \alpha-a)\left(B^{2}+\right.\right.\right.$ $\left.4 C-4 A C)) / 8 n^{2}\right)-\left((3 b(2 n+1)) /\left(8 l(n+1)^{2}\right)\right)-\gamma-a k^{2}$, where $A, B, C, a, b, \alpha$, and $\gamma$ are free parameters.

Substituting these solutions into (33), using (14) and (31), we obtain the traveling wave solution as follows:

$$
\begin{aligned}
u(t, x)= & {\left[-\frac{2 n+1}{4 l(n+1)} \pm \frac{B}{4 n(A-1)} \sqrt{\frac{(2 n+1)(2 \alpha-a)(A-1)^{2}}{b l}}\right.} \\
& \left. \pm \frac{1}{2 n} \sqrt{\frac{(2 n+1)(2 \alpha-a)(A-1)^{2}}{b l}}\left(\frac{G^{\prime}(\xi)}{G(\xi)}\right)\right]^{1 / 2 n} e^{i\left(-k x+w\left(t^{\delta} / \delta\right)+\theta\right),} \\
u(t, x)= & {\left[-\frac{2 n+1}{4 l(n+1)} \pm \frac{B}{4 n C} \sqrt{\frac{(2 n+1)(2 \alpha-a) C^{2}}{b l}}\right.} \\
& \left. \pm \frac{1}{2 n} \sqrt{\frac{(2 n+1)(2 \alpha-a) C^{2}}{b l}}\left(\frac{G^{\prime}(\xi)}{G(\xi)}\right)^{-1}\right]^{1 / 2 n} e^{i\left(-k x+w\left(t^{\delta} / \delta\right)+\theta\right),}
\end{aligned}
$$

where $G^{\prime}(\xi) / G(\xi)$ is defined in Section 3, and $\xi=x+2 a k\left(t^{\delta}\right.$ $/ \delta)$. 


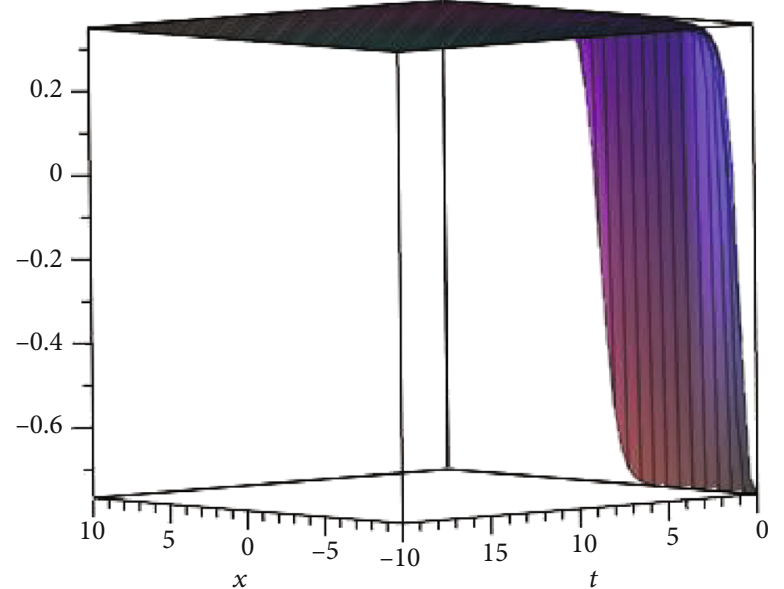

(a) $\delta=1 / 5$

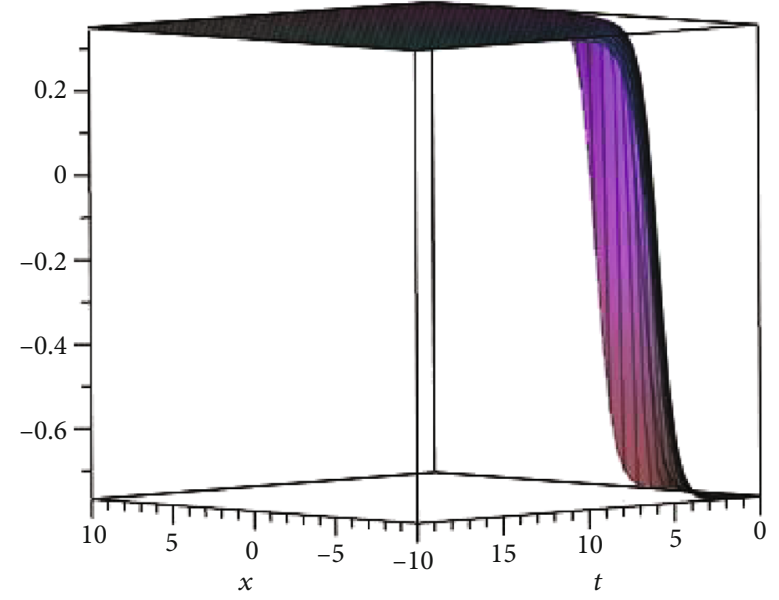

(b) $\delta=1 / 2$

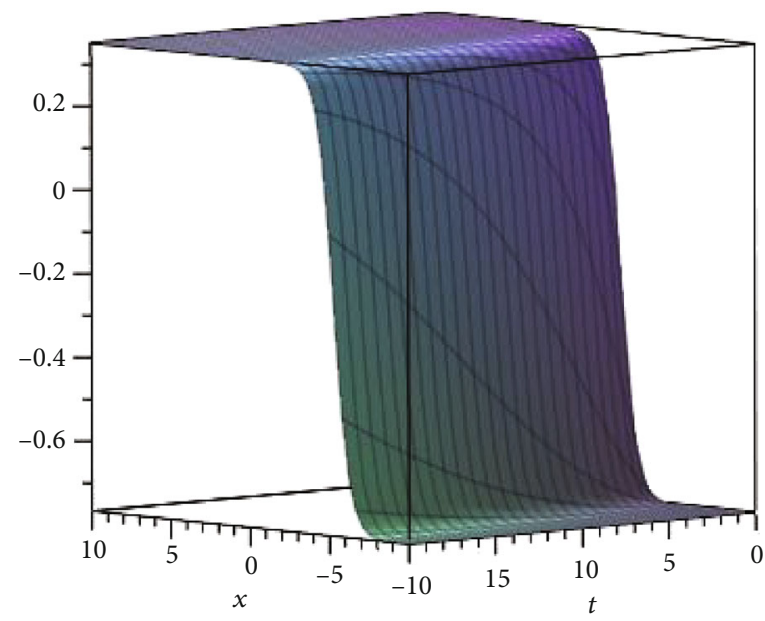

(c) $\delta=4 / 5$

FIgURE 5: The positive real part of $u_{1_{1}}(t, x)$ in Equation (37) for differential values of the fractional parameter $\delta$.

Family 1. The hyperbolic function solutions can be obtained as $B^{2}-4(A-1) C>0$ and $A \neq 1$. Then,

$$
\begin{aligned}
u_{1}(t, x)= & {\left[-\frac{2 n+1}{4 l(n+1)} \pm \frac{B}{4 n(A-1)} \sqrt{\frac{(2 n+1)(2 \alpha-a)(A-1)^{2}}{b l}}\right.} \\
& \pm \frac{1}{2 n} \sqrt{\frac{(2 n+1)(2 \alpha-a)(A-1)^{2}}{b l}} \\
& \left.\cdot\left(\frac{B}{2(1-A)}+\frac{\sqrt{B^{2}+4 C-4 A C}}{2(1-A)} H_{1}\right)\right]^{1 / 2 n} e^{i\left(-k x+w\left(t^{\delta} / \delta\right)+\theta\right),} \\
u_{2}(t, x)= & {\left[-\frac{2 n+1}{4 l(n+1)} \pm \frac{B}{4 n C} \sqrt{\frac{(2 n+1)(2 \alpha-a) C^{2}}{b l}}\right.} \\
& \pm \frac{1}{2 n} \sqrt{\frac{(2 n+1)(2 \alpha-a) C^{2}}{b l}} \\
& \left.\cdot\left(\frac{B}{2(1-A)}+\frac{\sqrt{B^{2}+4 C-4 A C}}{2(1-A)} H_{1}\right)^{-1}\right]^{1 / 2 n} e^{i\left(-k x+w\left(t^{\delta} / \delta\right)+\theta\right),}
\end{aligned}
$$

where $H_{1}=\left(C_{1} \sinh \left(\left(\sqrt{B^{2}+4 C-4 A C} / 2\right)\left(x+2 a k\left(t^{\delta}\right)\right.\right.\right.$ $\left.\delta)))+C_{2} \cosh \left(\left(\sqrt{B^{2}+4 C-4 A C} / 2\right)\left(x+2 a k\left(t^{\delta} / \delta\right)\right)\right)\right) /\left(C_{1}\right.$ $\cosh \left(\left(\sqrt{B^{2}+4 C-4 A C} / 2\right)\left(x+2 a k\left(t^{\delta} / \delta\right)\right)+C_{2} \sinh ((\right.$ $\left.\left.\left.\sqrt{B^{2}+4 C-4 A C} / 2\right)\left(x+2 a k\left(t^{\delta} / \delta\right)\right)\right)\right)$, and $C_{1}$ and $C_{2}$ are arbitrary constants.

In particular, if we assume that $C_{2}=0, A>1$, and $C_{1} \neq 0$ in (35), then we have

$$
\begin{aligned}
u_{1_{1}}(t, x)= & {\left[-\frac{2 n+1}{4 l(n+1)} \mp \frac{1}{4 n} \sqrt{\frac{(2 n+1)(2 \alpha-a)\left(B^{2}+4 C-4 A C\right)}{b l}} \tanh \right.} \\
& \left.\cdot\left(\frac{\sqrt{B^{2}+4 C-4 A C}}{2}\left(x+2 a k \frac{t^{\delta}}{\delta}\right)\right)\right]^{1 / 2 n} e^{i\left(-k x+w\left(t^{\delta} / \delta\right)+\theta\right)},
\end{aligned}
$$

and if $C_{1}=0, A>1$, and $C_{2} \neq 0$ in (35), then we obtain

$$
\begin{aligned}
u_{1_{2}}(t, x)= & {\left[-\frac{2 n+1}{4 l(n+1)} \mp \frac{1}{4 n} \sqrt{\frac{(2 n+1)(2 \alpha-a)\left(B^{2}+4 C-4 A C\right)}{b l}}\right. \text { coth }} \\
& \left.\cdot\left(\frac{\sqrt{B^{2}+4 C-4 A C}}{2}\left(x+2 a k \frac{t^{\delta}}{\delta}\right)\right)\right]^{1 / 2 n} e^{i\left(-k x+w\left(t^{\delta} / \delta\right)+\theta\right)} .
\end{aligned}
$$




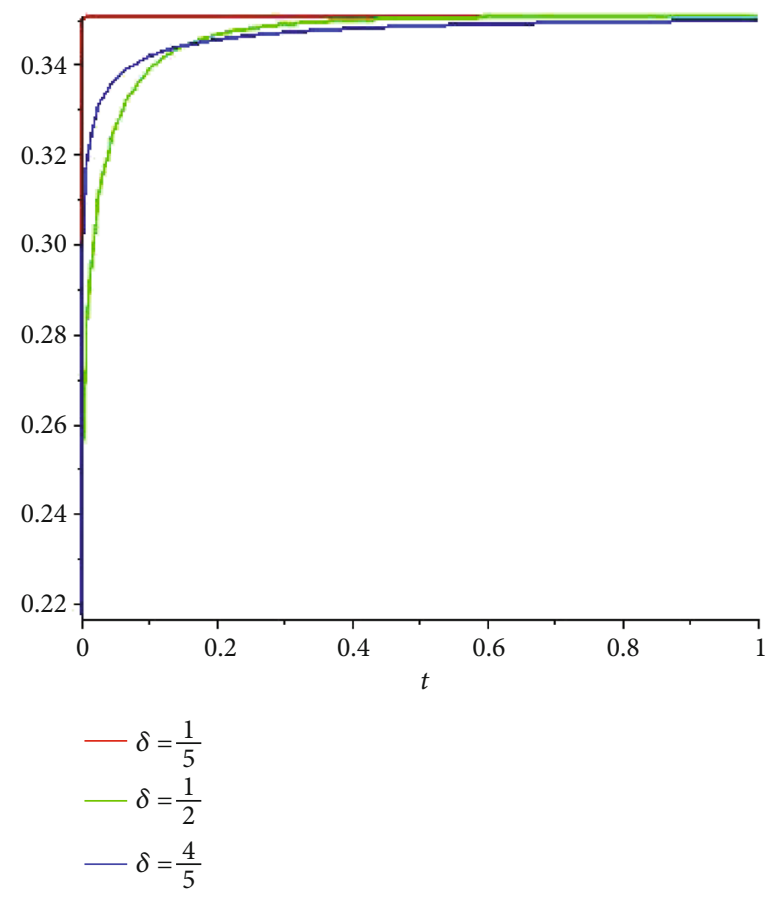

FIGURE 6: The positive real part of $u_{1_{1}}(t, x)$ at $x=1.0$ in Equation (37) for differential values of the fractional parameter $\delta$

The fixed values are $\alpha=2, a=1, b=1, A=2, B=4, C=3$, $k=1, l=3$, and $n=2$, and the fractional parameter $\delta \in\{1 / 5$ $, 1 / 2,1\}$ is used to plot the real part of the exact solution $u_{1_{1}}$ $(t, x)$ as in Figures 5 and 6.

Family 2. The trigonometric function solutions can be obtained as $4(A-1) C-B^{2}>0$ and $A \neq 1$. Then,

$$
\begin{aligned}
u_{3}(t, x)= & {\left[-\frac{2 n+1}{4 l(n+1)} \pm \frac{B}{4 n(A-1)} \sqrt{\frac{(2 n+1)(2 \alpha-a)(A-1)^{2}}{b l}}\right.} \\
& \pm \frac{1}{2 n} \sqrt{\frac{(2 n+1)(2 \alpha-a)(A-1)^{2}}{b l}} \\
& \left.\cdot\left(\frac{B}{2(1-A)}+\frac{\sqrt{4 A C-4 C-B^{2}}}{2(1-A)} H_{2}\right)\right]^{1 / 2 n} e^{i\left(-k x+w\left(t^{\delta} / \delta\right)+\theta\right)} \\
u_{4}(t, x)= & -\frac{2 n+1}{4 l(n+1)} \pm \frac{B}{4 n C} \sqrt{\frac{(2 n+1)(2 \alpha-a) C^{2}}{b l}} \\
& \pm \frac{1}{2 n} \sqrt{\frac{(2 n+1)(2 \alpha-a) C^{2}}{b l}} \\
& \left.\cdot\left(\frac{B}{2(1-A)}+\frac{\sqrt{4 A C-4 C-B^{2}}}{2(1-A)} H_{2}\right)^{-1}\right]^{1 / 2 n} e^{i\left(-k x+w\left(t^{\delta} / \delta\right)+\theta\right)}
\end{aligned}
$$

where $H_{2}=\left(-C_{1} \sin \left(\left(\sqrt{4 A C-B^{2}-4 C} / 2\right)\left(x+2 a k\left(t^{\delta} /\right.\right.\right.\right.$ $\left.\delta)))+C_{2} \cos \left(\left(\sqrt{4 A C-B^{2}-4 C} / 2\right)\left(x+2 a k\left(t^{\delta} / \delta\right)\right)\right)\right) /\left(C_{1}\right.$ $\cos \left(\left(\sqrt{4 A C-B^{2}-4 C} / 2\right)\left(x+2 a k\left(t^{\delta} / \delta\right)\right)\right)+C_{2} \sin (($ $\left.\left.\left.\sqrt{4 A C-B^{2}-4 C} / 2\right)\left(x+2 a k\left(t^{\delta} / \delta\right)\right)\right)\right)$, and $C_{1}$ and $C_{2}$ are arbitrary constants.
Specifically, if we suppose that $C_{2}=0, A>1$, and $C_{1} \neq 0$ in (39), then we obtain

$$
\begin{aligned}
u_{3_{1}}(t, x)= & {\left[-\frac{2 n+1}{4 l(n+1)} \pm \frac{1}{4 n} \sqrt{\frac{(2 n+1)(2 \alpha-a)\left(4 A C-4 C-B^{2}\right)}{b l}}\right.} \\
& \left.\cdot \tan \left(\frac{\sqrt{4 A C-4 C-B^{2}}}{2}\left(x+2 a k \frac{t^{\delta}}{\delta}\right)\right)\right]^{1 / 2 n} e^{i\left(-k x+w\left(t^{\delta} / \delta\right)+\theta\right),}
\end{aligned}
$$

and if $C_{1}=0, A>1$, and $C_{2} \neq 0$ in (39), then we obtain

$$
\begin{aligned}
u_{3_{2}}(t, x)= & {\left[-\frac{2 n+1}{4 l(n+1)} \mp \frac{1}{4 n} \sqrt{\frac{(2 n+1)(2 \alpha-a)\left(4 A C-4 C-B^{2}\right)}{b l}}\right.} \\
& \left.\cdot \cot \left(\frac{\sqrt{4 A C-4 C-B^{2}}}{2}\left(x+2 a k \frac{t^{\delta}}{\delta}\right)\right)\right]^{1 / 2 n} e^{i\left(-k x+w\left(t^{\delta} / \delta\right)+\theta\right)} .
\end{aligned}
$$

The fixed values are $\alpha=2, a=1, b=1, A=4, B=3, C=3$, $k=1, l=3$, and $n=2$, and the fractional parameter $\delta \in\{1 / 5$, $1 / 2,1\}$ is used to plot the real part of the exact solution $u_{3_{1}}$ $(t, x)$ as in Figures 7 and 8.

Family 3. The rational function solutions can be obtained as $B^{2}-4(A-1) C=0$ and $A \neq 1$. Then,

$$
\begin{aligned}
u_{5}(t, x)= & {\left[-\frac{2 n+1}{4 l(n+1)} \pm \frac{B}{4 n(A-1)} \sqrt{\frac{(2 n+1)(2 \alpha-a)(A-1)^{2}}{b l}}\right.} \\
& \pm \frac{1}{2 n(1-A)} \sqrt{\frac{(2 n+1)(2 \alpha-a)(A-1)^{2}}{b l}} \\
& \left.\cdot\left(\frac{C_{1}}{C_{1} \xi+C_{2}}+\frac{B}{2}\right)\right]^{1 / 2 n} e^{i\left(-k x+w\left(t^{\delta} / \delta\right)+\theta\right)},
\end{aligned}
$$




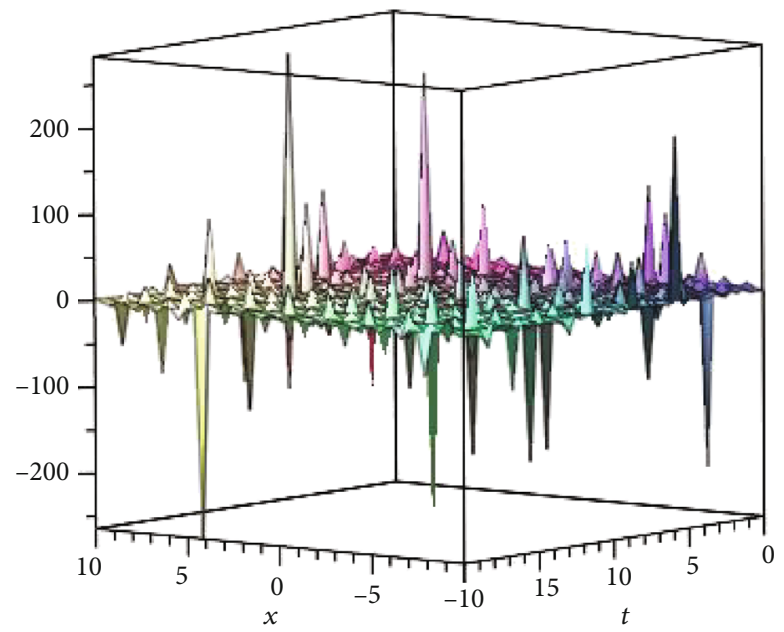

(a) $\delta=1 / 5$

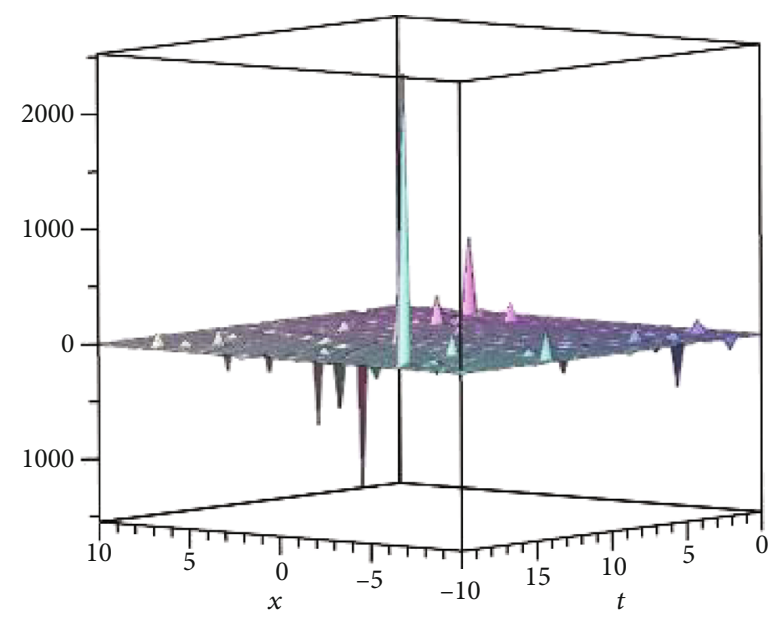

(b) $\delta=1 / 2$

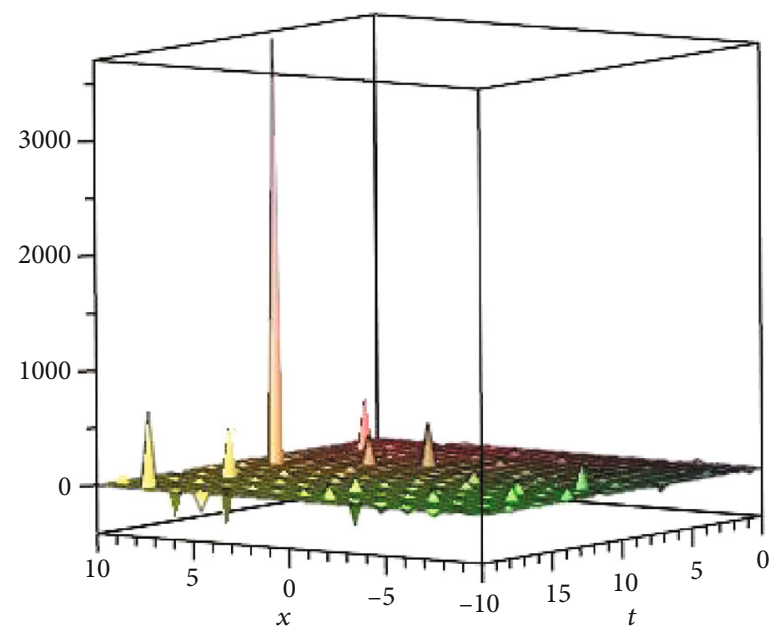

(c) $\delta=4 / 5$

FIgURE 7: The positive real part of $u_{3_{1}}(t, x)$ in Equation (41) for differential values of the fractional parameter $\delta$. 


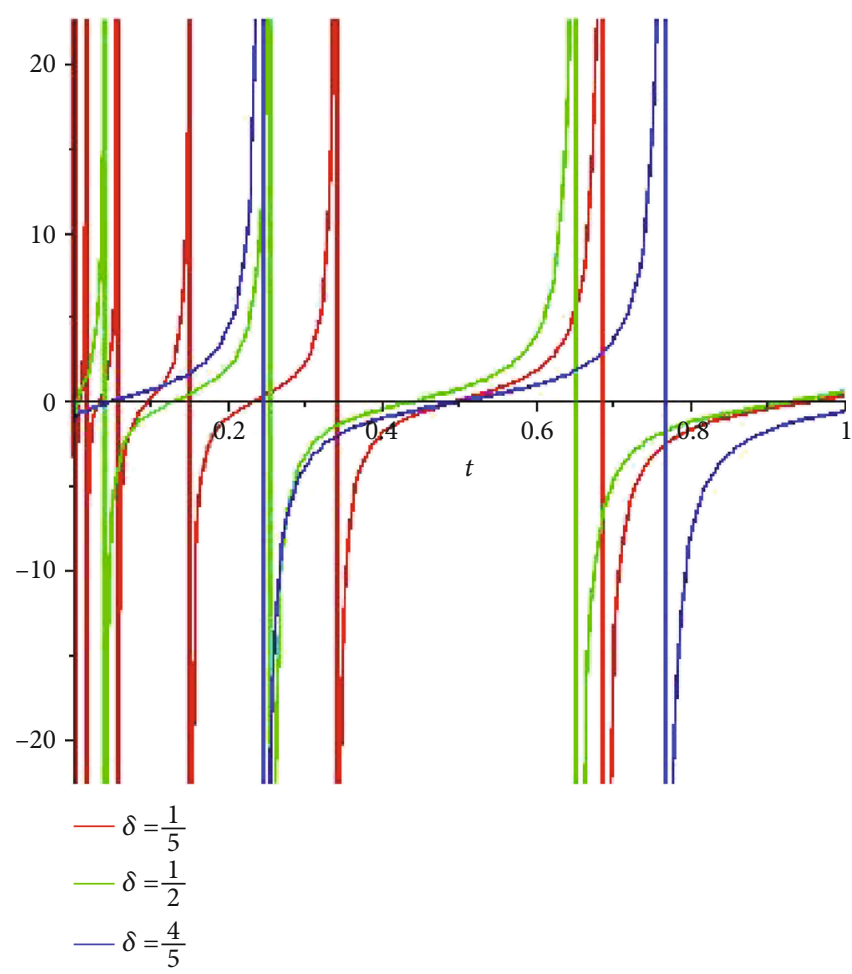

FIGURE 8: The positive real part of $u_{3_{1}}(t, x)$ at $x=1.0$ in Equation (41) for differential values of the fractional parameter $\delta$

$$
\begin{aligned}
u_{6}(t, x)= & {\left[-\frac{2 n+1}{4 l(n+1)} \pm \frac{B}{4 n C} \sqrt{\frac{(2 n+1)(2 \alpha-a) C^{2}}{b l}}\right.} \\
& \pm \frac{1}{2 n(1-A)} \sqrt{\frac{(2 n+1)(2 \alpha-a) C^{2}}{b l}} \\
& \left.\cdot\left(\frac{C_{1}}{C_{1} \xi+C_{2}}+\frac{B}{2}\right)^{-1}\right]^{1 / 2 n} e^{i\left(-k x+w\left(t^{\delta} / \delta\right)+\theta\right)}
\end{aligned}
$$

\section{Conclusion}

The time fractional complex GL equation with the Kerr law and dual-power law nonlinearity, which depicts the dynamics of soliton propagation through optical fibers over longer distances, is studied by using the extended $\left(G^{\prime} / G\right)$-expansion method. A series of new exact solutions are constructed, including hyperbolic function solutions, trigonometric function solutions, and rational function solutions. Comparing our results with the solutions in the previous literature, we get many new exact solutions. In order to further understand the dynamic behaviors of these results, with the help of software Maple 18, we have provided some graphical representation of the exact solutions of the time fractional complex GL equation with the Kerr law and dual-power law nonlinearity by setting the appropriate parameters. We believe that the obtained solutions may be of cardinal significance in the field of nonlinear optics. Moreover, in the paper, we have confirmed that the extended $\left(G^{\prime} / G\right)$-expansion method is powerful, reliable, and systematic, and they could be applied for solving more extensive and complicated fractional partial differential equations.

\section{Data Availability}

No data were used to support this study.

\section{Conflicts of Interest}

The authors declare that there is no conflict of interest regarding the publication of this paper.

\section{Acknowledgments}

This work was supported by the Scientific Research Funds of Chengdu University under grant no. 2081920034.

\section{References}

[1] A. Elmandouh, "Bifurcation and new traveling wave solutions for the 2D Ginzburg-Landau equation," The European Physical Journal Plus, vol. 135, no. 8, pp. 648-662, 2020.

[2] A. Biswas and R. T. Alqahtani, "Optical soliton perturbation with complex Ginzburg-Landau equation by semi-inverse variational principle," Optik, vol. 147, pp. 77-81, 2017.

[3] A. Biswas, Y. Yildirim, E. Yasar et al., "Optical soliton perturbation with complex Ginzburg-Landau equation using trial solution approach,” Optik, vol. 160, pp. 44-60, 2018.

[4] W. J. Corrêa and T. Özsari, "Complex Ginzburg-Landau equations with dynamic boundary conditions," Nonlinear Anaysis: Real World Applications, vol. 41, pp. 607-641, 2018. 
[5] M. Mirzazadeh, M. Erici, A. Sonmezoglu et al., "Optical solitons with complex Ginzburg-Landau equation," Nonlienar Dyanmical, vol. 85, no. 3, pp. 1979-2016, 2016.

[6] V. López, "Numerical continuation of invariant solutions of the complex Ginzburg-Landau equation," Communications in Nonlinear Science and Numerical Simulation, vol. 61, pp. 248-270, 2018.

[7] S. Arshed, A. Biswas, F. Mallawi, and M. R. Belic, "Optical solitons with complex Ginzburg-Landau equation having three nonlinear forms," Physics Letters A, vol. 383, no. 36, article 12026, 2019.

[8] V. F. Morales-Delgado, J. F. Gómez-Aguilar, M. A. TanecoHernández, and D. Baleanu, "Modeling the fractional nonlinear Schrödinger equation via Liouville-Caputo fractional derivative," Optik, vol. 162, pp. 1-7, 2018.

[9] V. F. Morales-Delgado, J. F. Gómez-Aguilar, and D. Baleanu, "A new approach to exact optical soliton solutions for the nonlinear Schrödinger equation," The European Physical Journal Plus, vol. 133, no. 5, article 189, 2018.

[10] H. Yépez-Martínez, J. F. Gómez-Aguilar, and A. Atangana, "First integral method for non-linear differential equations with conformable derivative," Mathematical Modelling of Natural Phenomena, vol. 13, no. 1, article 14, 2018.

[11] H. Yépez-Martínez, J. F. Gómez-Aguilar, and D. Baleanu, "Beta-derivative and sub-equation method applied to the optical solitons in medium with parabolic law nonlinearity and higher order dispersion," Optik, vol. 155, pp. 357-365, 2018.

[12] H. Yépez-Martínez and D. Baleanu, "Schrödinger equation involving fractional operators with non-singular kernel," Journal of Electromagnetic Waves and Applications, vol. 31, no. 7, pp. 752-761, 2017.

[13] B. Ghanbari and H. Yépez-Martínez, "New exact optical soliton solutions for nonlinear Schrödinger equation with second-order spatio-temporal dispersion involving M-derivative," Modern Physics Letters B, vol. 33, no. 20, article 1950235, 2019.

[14] H. Yépez-Martínez and J. F. Gómez-Aguilar, "M-derivative applied to the dispersive optical solitons for the SchrödingerHirota equation," The European Physical Journal Plus, vol. 134, no. 3, pp. 1-11, 2019.

[15] H. Yépez-Martínez and J. F. Gómez-Aguilar, "M-derivative applied to the soliton solutions for the Lakshmanan "CPorsezian"CDaniel equation with dual-dispersion for optical fibers," Optical and Quantum Electronics, vol. 51, no. 1, article 31, 2019.

[16] G. Akram and N. Mahak, "Application of the first integral method for solving $(1+1)$ dimensional cubic-quintic complex Ginzburg-Landau equation," Optik, vol. 164, pp. 210-217, 2018.

[17] J. B. Li and J. P. Shi, "Bifurcations and exact solutions of acdriven complex Ginzburg-Landau equation," Applied Mathematics and Computation, vol. 221, pp. 102-110, 2013.

[18] H. Rezazadeh, "New solitons solutions of the complex Ginzburg-Landau equation with Kerr law nonlinearity," Optik, vol. 167, pp. 218-227, 2018.

[19] E. Yomba and G. A. Zakeri, "Exact solutions in nonlinearly coupled cubic-quintic complex Ginzburg-Landau equations," Physics Letters A, vol. 377, no. 3-4, pp. 148-157, 2013.

[20] K. W. Chuang and Y. Y. Cao, "Exact front, soliton and hole solutions for a modified complex Ginzburg-Landau equation from the harmonic balance method," Applied Mathematics and Computation, vol. 218, no. 9, pp. 5140-5145, 2012.

[21] P. D. Wang and C. M. Huang, "An implicit midpoint difference scheme for the fractional Ginzburg-Landau equation," Journal of Computational Physics, vol. 312, pp. 31-49, 2016.

[22] N. Wang and C. M. Huang, "An efficient split-step quasicompact finite difference method for the nonlinear fractional Ginzburg-Landau equations," Computers \& Mathematics with Applications, vol. 75, no. 7, pp. 2223-2242, 2018.

[23] M. Li, C. M. Huang, and N. Wang, "Galerkin finite element method for the nonlinear fractional Ginzburg-Landau equation," Applied Numerical Mathematics, vol. 118, pp. 131-149, 2017.

[24] M. Zhang, G. F. Zhang, and L. D. Liao, "Fast iterative solvers and simulation for the space fractional Ginzburg-Landau equations," Computers \& Mathematics with Applications, vol. 78, no. 5, pp. 1793-1800, 2019.

[25] S. Arshed, "Soliton solutions of fractional complex GinzburgLandau equation with Kerr law and non-Kerr law media," Optik, vol. 160, pp. 322-332, 2018.

[26] N. Raza, "Exact periodic and explicit solutions of the conformable time fractional Ginzburg Landau equation," Optical and Quantum Electroncics, vol. 50, no. 3, pp. 154-170, 2018.

[27] M. A. Abdou, A. A. Soliman, A. Biswas, M. Ekici, Q. Zhou, and S. P. Moshokoa, "Dark-singular combo optical solitons with fractional complex Ginzburg-Landau equation," Optical, vol. 171, pp. 463-467, 2018.

[28] S. Sirisubtawee, S. Koonprasert, S. Sungnul, and T. Leekparn, "Exact traveling wave solutions of the space-time fractional complex Ginzburg-Landau equation and the space-time fractional Phi-4 equation using reliable methods," Advance in Differentce Equations, vol. 2019, no. 1, pp. 219-241, 2019.

[29] B. Ghanbari and J. F. Gómez-Aguilar, "Optical soliton solutions of the Ginzburg-Landau eqiaton with conformable derivative and Kerr law nonlinearity," Revista Mexicana de Fisica, vol. 65, pp. 73-81, 2019. 